\title{
PROGRAMAS Y EJERCICIOS DE FLEXIBILIDAD DENTRO DE LAS CLASES DE EDUCACIÓN FÍSICA, EN NIÑOS Y NIÑAS ESCOLARES, Y SU EFECTO EN LA MEJORA DE LA EXTENSIBILIDAD ISQUIOSURAL: UNA REVISIÓN SISTEMÁTICA
}

Soriano-Férriz, Beatriz; Alacid, Fernando

PROGRAMAS Y EJERCICIOS DE FLEXIBILIDAD DENTRO DE LAS CLASES DE EDUCACIÓN FÍSICA, EN NIÑOS Y NIÑAS ESCOLARES, Y SU EFECTO EN LA MEJORA DE LA EXTENSIBILIDAD ISQUIOSURAL: UNA REVISION SISTEMÁTICA

MHSalud, vol. 15, núm. 1, 2018

Universidad Nacional, Costa Rica

Disponible en: http://www.redalyc.org/articulo.oa?id=237056016001

DOI: https://doi.org/10.15359/mhs.15-1.1

Esta obra está bajo una Licencia Creative Commons Atribución-NoComercial-SinDerivar 3.0 Internacional. 


\title{
PROGRAMAS Y EJERCICIOS DE FLEXIBILIDAD DENTRO DE LAS CLASES DE EDUCACIÓN FÍSICA, EN NIÑOS Y NIÑAS ESCOLARES, Y SU EFECTO EN LA MEJORA DE LA EXTENSIBILIDAD ISQUIOSURAL: UNA REVISIÓN SISTEMÁTICA
}

\author{
FLEXIBILITY PROGRAMS AND EXERCISES WITHIN PHYSICAL EDUCATION CLASSES \\ FOR SCHOOLCHILDREN, AND THEIR EFFECT ON THE IMPROVEMENT OF HAMSTRING \\ EXTENSIBILITY: A SYSTEMATIC REVIEW \\ PROGRAMAS E EXERCÍCIOS DE FLEXIBILIDADE PARA CLASSES DE EDUCAÇÃO FÍSICA, EM \\ CRIANCAS DE IDADE ESCOLAR, E SEU EFEITO PARA MELHORAR A EXTENSIBILIDADE ISQUIO- \\ SURAL: UMA REVISÃO SISTEMÁTICA
}

Beatriz Soriano-Férriz [1]

Universidad Católica San Antonio Murcia, España

bea.sf.ef@gmail.com

(iD http://orcid.org/0000-0002-0299-5385

Fernando Alacid [2]

Universidad Católica San Antonio Murcia, España

falacid@ucam.edu

(iD http://orcid.org/0000-0001-6126-5289
DOI: https://doi.org/10.15359/mhs.15-1.1

Redalyc: http://www.redalyc.org/articulo.oa?

$\mathrm{id}=237056016001$

Recepción: 02 Noviembre 2017

Aprobación: 10 Abril 2018

\section{ReSUMEN:}

El objetivo del presente artículo científico fue considerar la literatura científica existente sobre los efectos que produce en los niños y niñas de escuela primaria la intervención de programas y ejercicios de estiramiento que forman parte de las clases de Educación Física, sobre la extensibilidad de la musculatura isquiosural. Se realizaron búsquedas sobre 5 bases de datos electrónicas en el período comprendido entre los años 2007-2017. Se encontraron 22 artículos y solo 12 fueron analizados pues cumplieron con los criterios de inclusión. De la búsqueda manual de las referencias bibliográficas, se añadieron 3 más, para un total de 15 artículos incluidos. Los resultados señalaron que es posible desarrollar la extensibilidad isquiosural en los escolares a través de programas y ejercicios de estiramiento en las clases de Educación Física. Las puntuaciones en las pruebas mejoran significativamente en los grupos experimentales, pero no en los grupos control. Los programas y ejercicios se pueden llevar a cabo en la fase de calentamiento y en la vuelta a la calma, siendo más efectivo en esta segunda fase. Un volumen de estiramiento entre 4-7 minutos y 2-4 clases por semana, obtienen mejoras estadísticamente significativas en los niveles de extensibilidad. Hasta 1 minuto de volumen provoca aumento. Tras 5 semanas de desentrenamiento los niveles disminuyen. Los profesionales de Educación Física deberían de incluir programas y ejercicios de estiramiento en sus clases para provocar una mejora de la extensibilidad isquiosural en escolares. El aumento del rango del movimiento tiene relación con la eficacia en la práctica deportiva, la disminución del dolor lumbar y alteraciones en el raquis y la prevención de lesiones.

Palabras clave: Educación Física, Escolares, Flexibilidad.

\section{Abstract:}

This scientific article aimed to consider the existing scientific literature on the effects that the programs and stretching exercises that are part of the Physical Education classes on the extensibility of the hamstring musculature produce in primary school children. We searched five electronic databases in the period 2007-2017. Twenty-two articles were found, only twelve were analyzed fulfilling the inclusion criteria. From the manual search of the bibliographic references, three more were added, with a total of fifteen articles included. The results indicated that it is possible to develop hamstring extensibility in school children through programs and stretching exercises in Physical Education classes. The scores on the tests improved significantly in the experimental groups, but

\section{Notas DE AUTOR}

[1] Máster Universitario en Investigación en Educación Física y Salud

[2] Doctor en Ciencias de la Actividad Física y el Deporte 
not in the control groups. The programs and the exercises can be carried out in the warm-up phase and in the return to calm, being more effective in this second phase. A stretch volume between 4-7 minutes and 2-4 classes per week obtain statistically significant improvements in levels of extensibility. Up to one minute of volume causes an increase. After five weeks of detraining, the levels decreased. Physical Education professionals should include programs and stretching exercises in their classes to improve hamstring extensibility in school children. The increase in the range of movement is related to the effectiveness of sports practice, the reduction of low back pain and alterations in the rachis, and the prevention of injuries.

KEYWORDS: physical education, schoolchildren, flexibility.

\section{Resumo:}

O objetivo deste trabalho científico foi considerar a literatura científica existente sobre os efeitos produzidos pela intervencao dos programas e exercícios de estiramento sobre a extensibilidad da musculatura ísquios-sural, que fazem parte das classes de Educação Física das criancas ensino fundamental. Foram pesquisados 5 bancos de dados eletrônicos no período de 2007-2017. Foram encontrados 22 artigos e apenas 12 foram analisados, pois cumpriram com os critérios de inclusão. Da busca manual das referências bibliográficas, acrescentaram-se 3 mais, para um total de 15 artigos incluídos. Os resultados assinalaram que é possível desenvolver a extensibilidade ísquios-sural aos estudantes através de programas e exercícios de estiramento nas classes de Educação Física. As pontuações em as provas melhoram significativamente nos grupos experimentais, mas não nos grupos de controle. Programas e exercícios podem ser realizados na fase de aquecimento e no retorno à calma, para ser mais eficaz nesta segunda fase. Um volume de estiramento entre 4-7 minutos e 2-4 classes por semana, obtêm melhoras estatisticamente significativas nos níveis de extensibilidade. Até 1 minuto de volume provoca aumento. Depois de 5 semanas sem treinar, os níveis diminuem. Os profissionais de Educação Física deveriam de incluir programas e exercícios de estiramento em suas classes para melhorar a extensibilidade ísquios-sural nos estudantes. O aumento do tempo do movimento está relacionado com eficácia na prática esportiva, a diminuição da dor lumbar, alterações no raquis e a prevenção de lesões.

Palavras-Chave: Educação Física, Escolares, Flexibilidade.

\section{INTRODUCCIÓN}

Todos realizamos de forma cotidiana movimientos que requieren que nuestras articulaciones, ligamentos, tendones y músculos sean extensibles para poder moverse eficazmente. La flexibilidad es una de las capacidades físicas que más beneficios aporta para la salud, por lo que puede considerarse básica para mantener

una condición física adecuada y conseguir una vida más activa y saludable.

El término flexibilidad se define como "la capacidad para desplazar una articulación o una serie de articulaciones a través de una amplitud de movimiento completo, sin restricciones ni dolor, influenciada por músculos, tendones, ligamentos, estructuras óseas, tejido graso, piel y tejido conectivo asociado" (Hernández Díaz, 2006), la extensibilidad correspondería a la capacidad que tiene un músculo determinado de elongarse.

La musculatura isquiosural se constituye en un grupo muscular biarticular formado por los músculos semimembranoso, semitendinoso y bíceps femoral. Se originan en la tuberosidad isquiática y van a la tibia (semimembranoso, semitendinoso) y al peroné (bíceps femoral). Es una musculatura muy rica en receptores sensibles al estiramiento. Su acción biarticular produce la flexión de la rodilla y la extensión de la cadera, lo cual se convierte en una musculatura esencial para la actividad física.

La extensibilidad de la musculatura isquiosural condiciona directamente la movilidad de la pelvis al realizar movimientos de flexión del tronco. Si la movilidad es reducida, la pelvis tiene poca capacidad de moverse hacia delante con lo que implica a la columna vertebral para hacer ese movimiento de flexión, pudiendo provocar alteraciones en el ritmo lumbar-pélvico (López-Miñarro, Muyor y Alacid, 2012) y la aparición de otras enfermedades como el dolor lumbar, las desalineaciones espinales sagitales o las hernias discales.

Hoy en día, se conoce que la extensibilidad isquiosural disminuye significativamente durante los años escolares (Kanásová, 2016 y Rodríguez et al., 2016). Es en esta edad donde se da el conocido "síndrome de isquiotibiales cortos", cuya causa no es conocida, pero implica una reducción muy importante de la extensibilidad isquiosural, por lo que se debe trabajar desde edades tempranas. Principalmente la adopción de malos hábitos posturales y el aumento de la conducta sedentaria tienen que ver con el acortamiento de 
la musculatura, por lo que la iniciación oportuna de un programa de ejercicios de estiramiento dentro de las clases de Educación Física en la escuela podría influir positivamente en la eliminación del acortamiento muscular.

El cuidado de los niños y las niñas, de su saludable desarrollo físico desde la temprana edad, es tarea principal no solo de padres, sino también de maestros y escuelas secundarias. Desafortunadamente, uno de cada cinco escolares tiene un nivel de extensibilidad en la musculatura isquiosural indicativo de riesgo para la salud (Castro Piñero et al., 2013).

La asignatura de Educación Física tiene como finalidad principal desarrollar en el alumno valores referenciados al cuerpo, al movimiento y a la relación con el entorno. Está vinculada a la adquisición de competencias relacionadas con la salud, la adquisición de hábitos responsables de actividad física regular y adopción de actitudes críticas ante prácticas sociales no saludables (R.D 126/2014, de 28 de febrero, por el que se establece el currículo básico de la Educación Primaria). La extensibilidad del músculo isquiosural se mejora significativamente cuando se realizan ejercicios de estiramientos de forma sistemática (Castro Piñero et al. 2013).

El marco escolar y el área de Educación Física son propicios para favorecer este ámbito de la extensibilidad muscular ya que el trabajo de estiramiento es propio de esta área. Los maestros y profesores deben de diseñar programas para el trabajo de la movilidad en sus clases. Bien es sabido que se encuentran con problemas a la hora del diseño tales como el tiempo, la frecuencia de las sesiones, los períodos vacacionales, a parte del hecho de que son muchos los contenidos curriculares que deben desarrollarse cada año académico (Sánchez Rivas et al. 2014; Mayorga-Vega, 2014b).

La Educación Física suele estar restringida por su limitada asignación de tiempo en el plan de estudios, restricción que se acentúa además cuando el número de sesiones de Educación Física a la semana se limita solo a dos, norma en la mayoría de países europeos (European Commission/EACEA/Eurydice, 2013). Los programas de actividad física para la Educación Física o las actividades deportivas extraescolares deben desarrollar la extensibilidad isquiosural (Kanasová, 2008; Sainz de Baranda et al. 2006; Santonja et al. 2007). Rodríguez et al. (2016) añaden que la clase de Educación Física debe ser un escenario ideal para la prevención del síndrome cortedad isquiosural ya que tiene el potencial de optimizar las condiciones ambientales y dar retroalimentación prolongada llegando a un gran porcentaje de la población.

Por lo tanto, y por todo lo descrito, el objetivo principal de esta revisión sistemática fue analizar los efectos que produce la aplicación de programas y ejercicios de estiramientos en la musculatura isquiosural dentro de las clases de Educación Física en escolares sanos de primaria.

\section{Metodología}

\section{- Estrategia de búsqueda}

Se procedió a buscar artículos en las siguientes cinco bases de datos electrónicas/ buscadores en línea, de los últimos diez años, es decir, período comprendido desde el año 2007 hasta agosto del año 2017: Web of Science (WOS), Google Scholar, Dialnet, Pubmed y Researchgate. Los términos de búsqueda utilizados se basaron en la combinación de las palabras clave: Physical Education, Hamstring, Extensibility Program, Schoolchildren, Flexibility y Primary. Dichos términos se combinaron con el operador boleano AND y se introducían entre comillas. En WOS, se utilizó Hamstring and Schoolchildren $(n=13)$, en Google Scholar Hamstring Programme and Physical Education and Primary School $(n=38)$; mientras que para Dialnet fueron términos en castellano, por un lado, Programa extensibilidad isquiosural $(n=7)$ y, por otro, Extensibilidad isquiosural y niños de primaria $(n=1)$. En Researchgate se utilizó Hamstring and Physical 
Education ( $n=69)$ y en Pubmed, Hamstring Program and Physical Education ( $n=41)$. Además, se hicieron búsquedas manuales en las listas de referencias de todos los artículos incluidos.

\section{- Criterios de selección}

Los criterios de selección para la identificación, clasificación e inclusión de los estudios que trataran sobre los efectos que producían programas y ejercicios de estiramientos en niños y niñas de primaria dentro de las clases de Educación Física sobre la mejora de la extensibilidad isquiosural fueron: (a) estudios con fechas de publicación en el período comprendido entre los años 2007-2017 (agosto), (b) que el programa de intervención estuviera desarrollado dentro de las clases de Educación Física, (c) estudios en los que los participantes fueran niños y niñas con edades comprendidas entre los 6-12 años (Etapa Primaria), (d) estudios que trataran sobre la extensibilidad isquiotibial y las mediciones fueran aceptadas en la literatura científica, por ejemplo, ensayos de elevación de la pierna recta, prueba de sentarse y alcanzar (sit-and-reach), toque del dedo del pie (toe-touch), (e) estudios con participantes aparentemente sanos, libres de trastornos ortopédicos (dolor, lesión, fracturas, cirugías), ni discapacidades físicas o mentales. No se impusieron restricciones en el idioma.

\section{- Estudios de calificación}

Para esta revisión, se recogieron los datos de los estudios que informaron sobre los efectos que provocaba en los niños y niñas de primaria incluir programas y ejercicios de estiramientos dentro de las clases de Educación Física. Se analizó la relación que había entre la musculatura en concreto a trabajar, en este caso la musculatura isquiosural, tras la aplicación de los programas y ejercicios de trabajo de flexibilidad y las puntuaciones en las pruebas, para comprobar si había una mejora de la extensibilidad de este grupo muscular, debido a la preocupación que lleva la cortedad de esta musculatura sobre los problemas que origina a nivel de la columna vertebral cada vez en edades más tempranas y la prevención de posibles dolores o lesiones, entre otros, en la edad adulta. De cada estudio que fue incluido en esta revisión, se registraron los siguientes datos: autores y año, tamaño de la muestra ( $\mathrm{n}$ ) y participantes, instrumentos de medición y evaluación (test), intervención llevada a cabo (duración, grupos control y grupo experimentales, tiempo de estiramiento, volumen e intensidad, frecuencia, batería de ejercicios) y efecto conseguido.

\section{Resultados}

\section{Descripción del estudio:}

La Figura 1 muestra un diagrama de flujo del proceso de selección del estudio. De los 169 resultados de búsqueda de literatura, 22 publicaciones potencialmente relevantes fueron identificadas y recuperadas para una evaluación más detallada. De esas 22 publicaciones, 10 estudios no cumplieron con los criterios de inclusión y fueron eliminados. De la búsqueda manual en las referencias bibliográficas incluidas en los artículos seleccionados, se rescataron 3 artículos, con lo cual, y finalmente, 15 fueron los estudios que se incluyeron en la presente revisión.

Las características generales de los estudios en los que se incluían programas y ejercicios de estiramiento isquiosural como parte de las clases de Educación Física en niños de primaria, se muestran en la Tabla 1. 


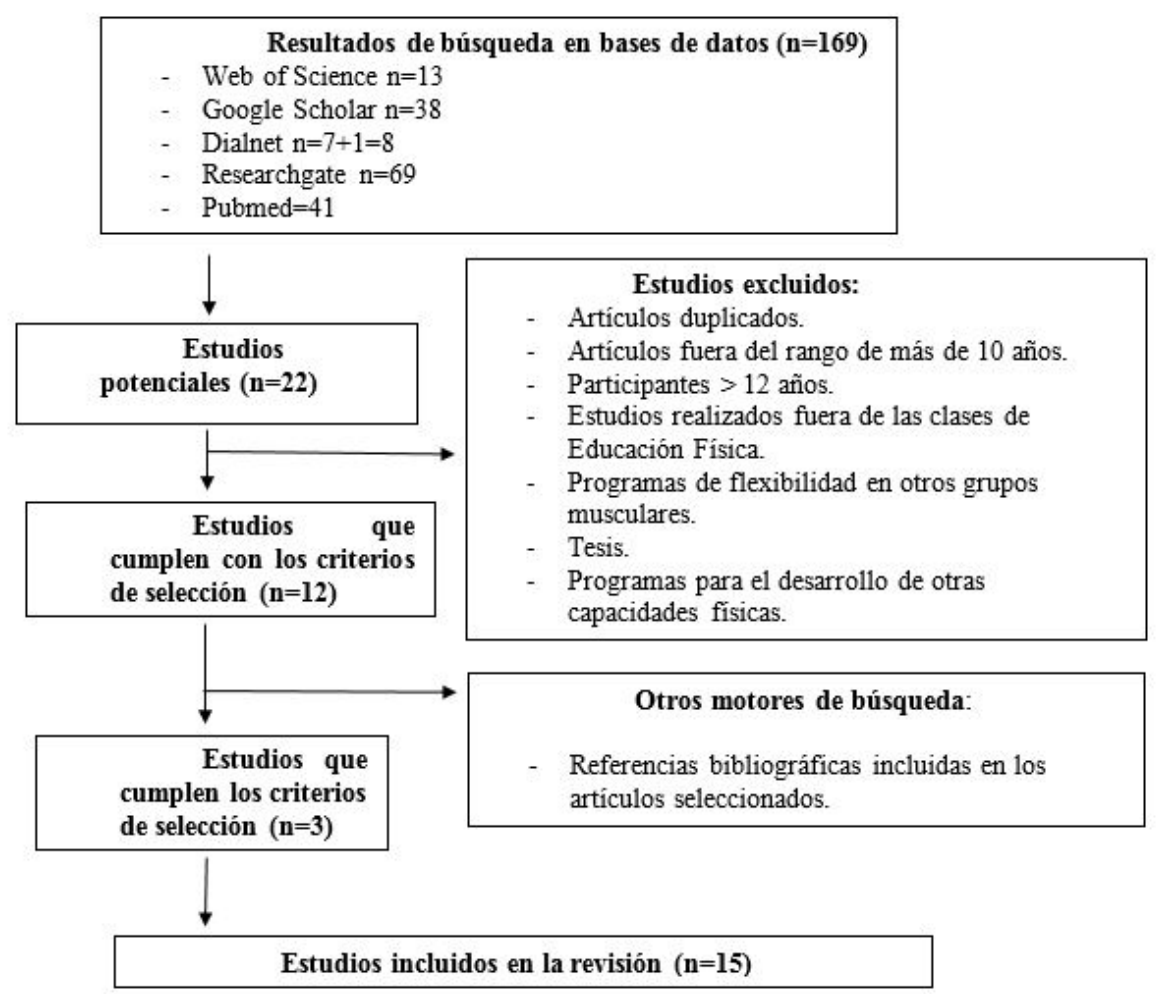

FIGURA 1.

Diagrama de flujo del proceso de selección de estudios.

Leyenda: $\mathrm{n}=$ número. 
TABLA 1.

Características generales de los estudios.

\begin{tabular}{|c|c|c|c|c|}
\hline Autores y año & Participantes & Test & Intervención & Efecto conseguido \\
\hline $\begin{array}{r}\text { Arruda } \\
(2012)\end{array}$ & $\begin{array}{l}\mathrm{n}=58(29 \text { niños, } 29 \\
\text { niñas }) \text { de } 8 \text { años }\end{array}$ & CSR & $\begin{array}{l}4 \text { grupos: GCM, GEM, GCF, GEF. } 16 \text { sema- } \\
\text { nas, } 2 \mathrm{vxw} \text {, método estático activo, } 6 \text { ejerci- } \\
\text { cios } 20 \text { s con } 10 \text { s de descanso. }\end{array}$ & $\begin{array}{l}\text { El estiramiento estático durante el calentamiento tuvo } \\
\text { un efecto crónico de mejorar la extensibilidad. }\end{array}$ \\
\hline $\begin{array}{l}\text { Kanásová } \\
(2008)\end{array}$ & $\begin{array}{l}\mathrm{n}=49 \text { niños/niñas de } \\
10-12 \text { años. }\end{array}$ & $\begin{array}{l}\text { A cada grupo muscular test } \\
\text { diferente. } 11 \text { grupos mus- } \\
\text { culares distintos: elevador } \\
\text { escápula, pectoral mayor, } \\
\text { iliopsoas, recto femoral, } \\
\text { tensor fascia lata, lumbar, } \\
\text { isquiotibiales, cuadrado } \\
\text { lumbar, triceps sural, erector } \\
\text { espinal. Norma y cortedad. }\end{array}$ & $\begin{array}{l}\text { GE, durante dos cursos. En el } 2^{\circ} \text { año de } \\
\text { experimentación, aplicación del programa. } \\
\text { ler año no se observa la aplicación de nin- } \\
\text { gún estímulo especial. }\end{array}$ & $\begin{array}{l}\text { Disminución de la cortedad muscular con la aplicación } \\
\text { de un programa de estiramientos a largo plazo en las } \\
\text { clases de EF. }\end{array}$ \\
\hline $\begin{array}{l}\text { Mayorga-Vega } \\
(2014 \mathrm{a})\end{array}$ & $\begin{array}{l}\mathrm{n}=73(36 \text { niños, } 37 \\
\text { niñas) de } 9 \text { y } 10 \\
\text { años. }\end{array}$ & CSR & $\begin{array}{l}3 \text { grupos: NTG, WUG, CDG. } 2 \mathrm{vxw} \text { durante } \\
10 \text { semanas, estiramientos pasivos estáticos } \\
\text { durante } 4 \text { min. }\end{array}$ & $\begin{array}{l}\text { Es posible desarrollar la extensibilidad isquiosural de } \\
\text { los escolares a través de un programa de estiramiento } \\
\text { en las clases de EF en las fases de calentamiento y } \\
\text { VC. }\end{array}$ \\
\hline $\begin{array}{l}\text { Mayorga-Vega } \\
(2014 \mathrm{~b})\end{array}$ & $\begin{array}{l}\mathrm{n}=45 \\
5-6 \text { años. }\end{array}$ & CSR & $\begin{array}{l}\text { GE durante las clases de } \mathrm{EF} 2 \mathrm{vxw} \text { durante } \\
8 \text { semanas. } 5 \text { semanas de desentrenamiento. } \\
\text { Técnica estática durante } 1 \text { min. en la VC } 4 \\
\text { ejercicios alternos, } 20 \text { s de mantenimiento. }\end{array}$ & $\begin{array}{l}\text { Aumento significativo de la puntuación SR ( } \mathrm{pc} \\
<0.001) \text {. Después de } 5 \text { semanas de desentrenamiento } \\
\text { la puntuación se vuelve a los niveles basales }(\mathrm{p}>0,05) \\
\text { aunque un programa de estiramiento de } 1 \mathrm{~min} \text {. parece } \\
\text { desarrollar la extensibilidad en los escolares. }\end{array}$ \\
\hline $\begin{array}{l}\text { Mayorga-Vega } \\
\text { (2014c) }\end{array}$ & $\begin{array}{l}\mathrm{n}=45(26 \text { niños } \mathrm{y} \\
19 \text { niñas) de } 10 \text { a } 11 \\
\text { años. }\end{array}$ & CSR & $\begin{array}{l}\text { GE durante las clases de EF } 2 \mathrm{vxw} \text { durante } \\
8 \text { semanas. } 5 \text { semanas de desentrenamiento. } \\
\text { Técnica estática. } 6 \text { min. en la VC. } 20 \text { s de } \\
\text { mantenimiento. } 6 \text { ejercicios distintos. }\end{array}$ & $\begin{array}{l}\text { La intervención del programa aumenta significativa- } \\
\text { mente la extensibilidad isquiosural y lumbar, aunque } \\
\text { después del período de desentrenamiento los niveles } \\
\text { de extensibilidad disminuyen, pero se mantienen más } \\
\text { altos que los niveles basales. }\end{array}$ \\
\hline $\begin{array}{l}\text { Mayorga-Vega } \\
(2016)\end{array}$ & $\begin{array}{l}\mathrm{n}=150(70 \text { niños } \mathrm{y} \\
80 \text { niñas }) \text { de entre } 7 \\
\text { y } 10 \text { años. }\end{array}$ & CSR & $\begin{array}{l}\mathrm{VC}, \mathrm{GE}, 42 \mathrm{vxw} 9 \text { semanas. } 5 \text { semanas de } \\
\text { desentrenamiento. } 2 \mathrm{vxw} \text { durante } 11 \text { semanas } \\
\text { GE1 4min., GE2 } 1 \text { min., Técnica estática, } \\
\text { 30s. }\end{array}$ & $\begin{array}{l}\text { Mejora de la extensibilidad isquiosural, así como man- } \\
\text { tenimiento de ganancias obtenidas después de progra- } \\
\text { ma de mantenimiento. No se encuentran diferencias } \\
\text { estadísticamente significativas entre los dos grupos. }\end{array}$ \\
\hline $\begin{array}{l}\text { Mayorga-Vega, } \\
\text { Merino-Marban } \\
\text { y García-Romero } \\
\text { (2013) }\end{array}$ & $\begin{array}{l}\mathrm{n}=72(40 \text { niños y } 32 \\
\text { niñas) de entre } 10- \\
12 \text { años. }\end{array}$ & $\begin{array}{l}\text { CSR } \\
\text { SRG } \\
\text { PSLR }\end{array}$ & $\begin{array}{l}\text { Estudio piloto con } 10 \text { estudiantes de prima- } \\
\text { ria para estimar validez. Tras calentamiento, } \\
\text { se realizan al azar las pruebas CSR, SRF Y } \\
\text { PSLR. Descanso pasivo de } 15 \mathrm{~min} \text {. entre las } \\
\text { pruebas. }\end{array}$ & $\begin{array}{l}\text { Se sugiere que la evaluación de la extensibilidad del } \\
\text { músculo isquiosural por la prueba SR debe realizarse } \\
\text { permitiendo flexión plantar. }\end{array}$ \\
\hline $\begin{array}{l}\text { Mayorga-Vega, } \\
\text { Merino-Marban, } \\
\text { Redondo-Martin } \\
\text { y Viciana } \\
\text { (2017) } \\
\end{array}$ & $\begin{array}{l}\mathrm{n}=37 \text { participantes } \\
(18 \text { niños y } 19 \text { ni- } \\
\text { ñas) de } 9 \text { años. }\end{array}$ & CSR & $\begin{array}{l}\text { GE: Estiramientos programados } 1 \mathrm{vxw} \text { du- } \\
\text { rante } 28 \text { semanas. Estiramientos estáticos } \\
\text { durante } 3 \text { min. en la VC, } 20 \text { s de manteni- } \\
\text { miento de la batería de ejercicios. }\end{array}$ & $\begin{array}{l}\text { Mejoras estadísticamente significativas en las puntua- } \\
\text { ciones SR }(p<0,01) \text {. }\end{array}$ \\
\hline $\begin{array}{l}\text { Mayorga-Vega, } \\
\text { Merino-Marban } \\
\text { y Sánchez-Rivas } \\
\text { (2014d) }\end{array}$ & $\begin{array}{l}\mathrm{n}=45(24 \text { niños } \mathrm{y} \\
21 \text { niñas }) \text { de } 9 \text { a } 10 \\
\text { años. }\end{array}$ & CSR & $\begin{array}{l}\text { GE, } 2 \text { vxw durante } 8 \text { semanas, desentrena- } \\
\text { miento de } 5 \text { semanas. Estiramientos está- } \\
\text { ticos } 5 \text { min. } 3 \text { series de } 4 \text { ejercicios. } 20 \text { s de } \\
\text { mantenimiento. }\end{array}$ & $\begin{array}{l}\text { Mejora la extensibilidad isquiosural de los escolares } \\
\text { con un programa de estiramiento; los efectos de mejo- } \\
\text { ra se pierden tras } 5 \text { semanas de desentrenamiento. }\end{array}$ \\
\hline $\begin{array}{l}\text { Rodríguez, } \\
\text { Santonja, López } \\
\text { Miñarro, Sáinz } \\
\text { de Baranda y } \\
\text { Yuste } \\
(2016) \\
\end{array}$ & $\begin{array}{l}\mathrm{n}=46 \text { ( } 20 \text { niños y } 26 \\
\text { niñas) de } 10 \text { años. } \\
\mathrm{n}=44 \text { ( } 21 \text { niños y } 23 \\
\text { niñas) de } 13 \text { años. }\end{array}$ & CSR & $\begin{array}{l}\text { GE, } 2 \text { vxw durante } 32 \text { semanas. Ejercicios } \\
\text { realizados al final del calentamiento } 3 \mathrm{~min} \text {. y } \\
\text { VC } 2 \text { min. con } 20 \text { s de mantenimiento. }\end{array}$ & $\begin{array}{l}\text { La puntuación aumentó tanto en los GE de secundaria } \\
(+7,22 \mathrm{~cm} ; \mathrm{p}<0,001) \text { como en los de primaria }(+1,95 \\
\mathrm{cm} ; \mathrm{p}>0,05) \text {. }\end{array}$ \\
\hline $\begin{array}{l}\text { Sánchez Rivas, } \\
\text { Mayorga-Vega, } \\
\text { Fernández Ro- } \\
\text { dríguez y Meri- } \\
\text { no-Marban } \\
\text { (2014) }\end{array}$ & $\begin{array}{l}\mathrm{n}=44(24 \text { niñas y } 20 \\
\text { niños) de } 7-8 \text { años. }\end{array}$ & CSR & $\begin{array}{l}\text { GE, } 9 \text { semanas, } 2 \mathrm{vxw} \text {. Una semana pre- } \\
\text { via y posterior se valoró la extensibilidad } \\
\text { con estiramientos durante } 3 \mathrm{~min} \text {. en la VC. } \\
\text { Técnica estático pasiva relajada, } 20 \mathrm{~s} \text { de } \\
\text { mantenimiento, } 3 \text { ejercicios, } 3 \text { series, } 5 \mathrm{~s} \text { de } \\
\text { descanso. }\end{array}$ & $\begin{array}{l}\text { Efecto positivo sobre los resultados del SR en escola- } \\
\text { res de Educación Primaria en la musculatura isquio- } \\
\text { sural. }\end{array}$ \\
\hline $\begin{array}{l}\text { Santonja, Sainz } \\
\text { de Baranda, } \\
\text { Rodríguez, } \\
\text { López Miñarro y } \\
\text { Canteras Jordana } \\
\text { (2007) }\end{array}$ & $\begin{array}{l}\mathrm{n}=62 \text { alumnos de } \\
10 \text { y } 11 \text { años. }\end{array}$ & PSLR & $\begin{array}{l}\text { GE1: estiramientos } 5 \mathrm{~min} .9 \text { meses } 2 \mathrm{vxw} \text {. } \\
\text { GE2: ejercicios de estiramiento } 5 \mathrm{~min} \text {. en } \mathrm{EF} \\
\text { y extraescolares, } 9 \text { meses, } 4 \mathrm{vxw} \text {. } \\
\text { ROM medido } 2 \mathrm{vxw} \text {, ejercicios mantenidos } \\
\text { 20s. }\end{array}$ & $\begin{array}{l}\text { Mejoras significativas en el ROM en los dos GE } \\
(p<0.001) \text {. Cuatro días por semana produjo una mayor } \\
\text { tasa de ganancias en el ROM }\left(16.9^{\circ}\right) \text { que dos días por } \\
\text { semana }\left(9.3^{\circ}\right) \text {. Para todos los grupos el ROM inicial y } \\
\text { final entre lados izquierdo y derecho fueron similares. }\end{array}$ \\
\hline
\end{tabular}

\section{Discusión}

Durante los años escolares la flexibilidad disminuye hasta el inicio de la pubertad. La disminución puede estar relacionada con el aumento de músculos y tendones y la rigidez que se produce alrededor de la articulación debido al crecimiento más rápido de los huesos. También se relaciona con la postura de sentado prolongada en la escuela y un estilo de vida sedentario. 
Los resultados de la presente revisión indicaron que la incorporación de programas y ejercicios de estiramientos dentro de las clases de Educación Física produce en los escolares de ambos sexos una mejora significativa en las puntuaciones en los diferentes test utilizados: clásico sit-and-reach (CSR), toe-touch (TT), elevación de la pierna recta (PSLR) y back-saver-sit (SRS), en los grupos experimentales, pero no en los grupos control.

También podría influir en estos puntajes las proporciones antropométricas de los miembros superior e inferior de los escolares. El CSR fue modificado por el SRF introduciéndose una flexión plantar, siendo esta modificación más alta en puntajes que el CSR, pero los valores de validez encontrados son moderados en ambas pruebas. Mientras tanto, Hartman y Looney (2003) estimaron la validez del back-saver SR, prueba que se creó para proteger la espalda de la tensión que se produce cuando el individuo realiza una flexión del tronco como en el CSR (Cooper Institute for Aerobics Research, 2004).

La frecuencia de la incorporación de programas y ejercicios de estiramiento podría tener relación positiva con el tamaño del efecto de intervención. Puesto que la Educación Física en muchos países europeos está limitada a dos sesiones por semana (Comisión Europea/EACEA/ Eurydice, 2013), la aplicación de programas de estiramiento con una frecuencia más alta no sería posible. En los estudios realizados solo 2 veces por semana, se demuestra que un estiramiento basado en un programa de desarrollo en Educación Física mejora la extensibilidad de la musculatura isquiosural. Merino Marban et al. (2015) indicaron que el tiempo activo aumentado para el aprendizaje en los períodos extracurriculares representaría una estrategia para los profesores de Educación Física en la mejora de la movilidad.

Mayorga-Vega et al. (2015) encontraron que tanto un programa de estiramiento realizado una vez a la semana como un programa realizado dos sesiones por semana mejora la extensibilidad isquiosural en los escolares de ambos sexos. No se encontraron diferencias estadísticamente significativas entre los dos grupos experimentales $(p>0,05)$. La duración del programa de intervención también parece ser un factor importante, la mayoría de estudios realizan programas de entre 8 y 32 semanas y logran mejoras en la extensibilidad isquiosural.

Un obstáculo que se encontró son los periodos vacacionales de desentrenamiento, donde disminuyen las ganancias de extensibilidad. La información científica actual es todavía escasa. La mayoría de los estudios encuentran que tras un período de desentrenamiento los niveles de extensibilidad disminuyen, incluso en algunos estudios disminuyen hasta alcanzar los niveles basales (Merino-Marban et al, 2014b).

En relación con la duración de cada ejercicio, observamos que en todos los estudios lograron mejoras en la musculatura isquiosural cuando esta fue estirada durante 20 segundos, manteniendo el ejercicio sin llegar a sentir dolor (Mayorga-Vega, 2014a, 2014c, 2014d; Mayorga-Vega et al., 2017; Rodríguez et al., 2016 y Santonja, 2007). Por lo tanto, esta intensidad media/baja podría ser eficaz para la mejora de la extensibilidad isquiosural.

Con respecto al volumen total de estiramiento, Santonja et al. (2007) y Arruda (2012) demostraron que cuando los músculos se estiran entre 1 y 7 minutos hay mejoras significativas en la extensibilidad de la musculatura isquiosural.

Las técnicas que se utilizan para realizar los estiramientos son técnicas activas y pasivas. Parece ser que las técnicas pasivas estáticas son principalmente recomendadas en los escolares ya que controlan mejor la alineación del raquis.

También los programas de estiramientos se pueden llevar a cabo en las clases de Educación Física tanto en la fase de calentamiento como en la vuelta a la calma (Mayorga-Vega, 2014a). Estudios como el de Arruda (2012) mencionan que los ejercicios de estiramientos realizados en la fase de vuelta a la calma surten más efectividad que en la fase de calentamiento, ya que en esta fase afecta negativamente a factores de fuerza explosiva, velocidad o habilidades deportivas. Aunque si el objetivo de la sesión fuera el desarrollo de la extensibilidad, no habría problema en realizarlo en la fase de calentamiento, pero es mejor en la fase de la vuelta a la calma. 
Por lo general, podemos decir que todos los estudios advierten que la inclusión de un programa de estiramiento como parte de las sesiones de Educación Física, sobre todo en la fase de vuelta a la calma por su mayor efectividad, provocan una mejora estadísticamente significativa en la extensibilidad isquiosural de los escolares.

La duración del programa está comprobada que con 8 semanas ya se obtienen mejoras en los resultados de las pruebas. La frecuencia con la que se realice el programa influye, cuanto mayor sea el número de sesiones, mejores resultados se esperan, ya que se ha visto que con las sesiones semanales curriculares y extracurriculares se obtienen mejores resultados que solo con las curriculares.

Bien es verdad que existen estudios que nos señalan que con solo una sesión por semana ya sería efectiva la extensibilidad en los escolares, utilizando esta estrategia para el diseño de programas cortos y que no se vea alterada la amplitud de contenidos que se han de tratar en el área. Todos los estudios son coincidentes en que la intensidad de mantenimiento del estiramiento se marca en veinte segundos, cifra válida para obtener resultados.

A pesar de las limitaciones que presenta, se encontró que el clásico sit-and-reach es la prueba lineal más utilizada en los estudios de evaluación de la extensibilidad isquiosural en escolares.

Ahora bien, y después de todos estos factores, cabe señalar que tras cinco semanas de desentrenamiento los valores obtenidos se pierden, incluso hasta los niveles basales.

\section{Conclusiones}

Los escolares que mantuvieron un programa de estiramiento como parte de las clases de Educación Física obtuvieron una mejora estadísticamente significativa en la extensibilidad de la musculatura isquiosural. Sin embargo, tras un período de desentrenamiento se observa una disminución de estas mejoras.

La frecuencia del programa y la duración del mismo parece tener relación positiva con el efecto producido. Los estudios seleccionados y analizados mostraron que la aplicación de programas cortos podría ser adecuado para facilitar a los maestros y profesores la organización de contenidos y obtener además mejoras en la extensibilidad isquiosural.

Los programas de estiramientos se pueden incluir tanto en la fase de calentamiento como en la vuelta a la calma, aunque se ha demostrado que al realizarlos dentro de la vuelta a la calma se obtiene una leve mejoría en el estiramiento, con lo cual puede ser más eficaz.

Las técnicas estáticas pasivas y activas se recomiendan principalmente en los escolares ya que contribuyen a un mayor control de la alineación del raquis.

\section{IMPLICACIONES PRÁCTICAS}

Los escolares que mantengan un estiramiento durante 20 segundos en cada ejercicio, usen un volumen entre 1 y 7 minutos por sesión y una frecuencia de $2-4$ clases por semana obtendrán mejoras estadísticamente significativas en los niveles de extensibilidad isquiosural.

\section{RECOMENDACIONES}

Los centros escolares deben promover hábitos de postura buscando el desarrollo musculoesquelético de los alumnos, además de en el área de Educación Física, dentro de toda la comunidad educativa, ya que la escuela es el escenario clave para la prevención y la mejora de la salud del alumno.

Los maestros y profesores de Educación Física deben incluir programas de estiramiento dentro de sus clases para obtener mejoras significativas en la extensibilidad isquiosural en los alumnos. La adecuación de 
programas de estiramiento cortos dentro de las clases de Educación Física sería una buena estrategia para trabajar la flexibilidad y obtener mejoras en la extensibilidad isquiosural, facilitando así el desarrollo de los contenidos del área.

Para que no baje el nivel de extensibilidad obtenida en los períodos vacacionales, se recomienda realizar estiramientos fuera del entorno escolar o en otros programas, dependiendo esto de la autonomía del alumno y la implicación de padres y tutores, además de añadir actividades extraescolares que mantengan y aumenten los niveles de extensibilidad muscular.

Se ha observado que el desempeño de un calentamiento antes de las mediciones de la prueba aumentaría los resultados de la prueba. Debido al orden de la evaluación, algunos escolares tendrían la medición inmediatamente después de terminar el calentamiento y otros lo harían pasados varios minutos, por lo tanto, para garantizar las mismas condiciones para todos es mejor no realizar ejercicios de calentamientos previos.

La técnica de estiramiento recomendada en los escolares coincide con la utilización de técnicas estáticas.

\section{REFERENCIAS}

Arruda, G. (2012). Chronic effect of static stretching performed during warm-up on flexibility in children. Brazilian Journal of Kinanthropometry and Human Performance, 296-304. doi: 10.5007/1980-0037.2012v14n3p296

Castro-Piñero, J., Girela-Rejón, M.J., González-Montesinos, J. L., Mora, J., Conde- Caveda, J., Sjöström, M., \& Ruiz, J. R. (2013) Percentile values for flexibility tests in youths aged 6 to 17 years: Influence of weight status. European Journal of Sport Science, 13 (2), 139-148.

Cooper Institute for Aerobics Research (2004). The Prudential Fitnessgram: Test administration manual (3rd ed). Champaign, IL: Human Kinetics.

European Commission/EACEA/Eurydice (2013). Physical Education and Sport at School in Europe Eurydice Report. Luxembourg: Publications Office of the European Union.

Hartman, J. G., \& Looney, M. (2003). Norm-Referenced and Criterion-Referenced Reliability and Validity of the Back-Saver Sit-and-Reach. Measurement in Physical Education and Exercise Science, 7(2), 71-87. doi: 10.1207/ S15327841MPEE0702_2

Hernández Díaz, P.E. (2006). Flexibilidad: Evidencia Científica y Metodología del Entrenamiento. Journal PubliCE. Recuperado de https://g-se.com/flexibilidad-evidencia-cientifica-y-metodología-del-entrenamiento-789-saa$\mathrm{S} 57 \mathrm{cfb} 27185532$

Kanásová, J. (2016). Reducing Shortened Muscles in 10-12-year-old Boys Through A Physical Exercise Programme. Medicina Sportiva. doi.org/10.2478/v10036-008-0022-x

López-Miñarro, P. A., Muyor, J. M., \& Alacid, F. (2012). Acute Effects of Hamstring Stretching on Sagittal Spinal Curvatures and Pelvic Tilt. Journal of Human Kinetics, 31, 69-78. doi.org/10.2478/v10078-012-0007-7

Mayorga-Vega, D., Merino-Marban, R., Garrido, F. J., \& Viciana, J. (2014a). Comparison between warm-up and cooldown stretching programs on hamstring extensibility gains in primary schoolchildren. Physical Activity Review, 2, 16-24.

Merino-Marban, R., Mayorga-Vega, D., Fernandez-Rodriguez, E., Vera-Estrada, F \& Viciana, J. (2014b). Effect of a physical education-based stretching programme on sit-and- reach score and its posterior European Physical Education Review. European Physical Education Review, 21(1), 83-92. doi.org/10.1177/1356336X14550942

Mayorga-Vega, D. (2014c). Effect of a short-term physical education based flexibility program on hamstring and lumbarextensibility and its posterior reduction in primary schoolchildren. Kinesiology, 227-233.

Mayorga-Vega, D. (2016). Effects of a Stretching Development and Maintenance Program on Hamstring Extensibility in Schoolchildren: A cluster-randomized controlled trial. Journal of Sports Science and Medicine, 15, 65-74.

Mayorga-Vega, D., Merino-Marban, R., \& García-Romero, J. C. (2013). Validity of sit-and-reach with plantar flexion test in children aged 10-12 years. Revista Internacional de Medicina Y Ciencias de La Actividad Física Y El Deporte, 15(59), 577-591. 
Mayorga-Vega, D., Merino-Marban R., Real J, Viciana J. (2015). A physical education-based stretching program performed once a week also improves hmastring extensibility in schoolchildren: a cluster-randomized controlled trial. Nutrición Hospitalaria, 32 (4), 1715-1721.

Mayorga-Vega, D., Merino-Marban, R., Redondo-Martín, F. J., \& Viciana, J. (2017). Effect of a one-session-perweek physical education-based stretching program on hamstring extensibility in schoolchildren. Kinesiology, 49, 101-108.

Mayorga-Vega, D., Merino-Marban, R., \& Sánchez-Rivas, E. (2014d) Effect of a short-term static stretching training program followed by five weeks of detraining on hamstring extensibility in children aged 9-10 years. Journal of Physical Education and Sport, 14(3), 355-359.

Mayorga-Vega, D., Merino-Marban, R., \& Viciana, J. (2014). Criterion-Related Validity of Sit-And-Reach Tests for Estimating Hamstring and Lumbar Extensibility: A Meta-Analysis. Journal of Sports Science and Medicine, 13, $1-14$.

Merino-Marban, R., Mayorga-Vega D., Fernandez-Rodriguez, E., Vera Estrada F., Viciana J. (2015). Effect of a physical education-based stretching programme on sit-and-reach score and its posterior reduction in elementary schoolchildren. European Phyisical Education Review, 21(1), 83-92.

Ministerio de Educación, Cultura y Deporte. (2014). Real Decreto 126 / 2014, de 28 de febrero, por el que se establece el currículo básico de la Educación Primaria. Boletín Oficial del Estado, 52, 19349-19420. Recuperado de https://www.boe.es/boe/dias/2014/03/01/pdfs/BOE-A-2014-2222.pdf.

Rodriguez, P.L.; Santonja, F.M.; López Miñarro, P.A.; Sáinz de Baranda, P.; Yuste, J. L. (2016). Mejora de la extensibilidad isquiosural tras un programa escolar de estiramientos. Science\&Sports, 23, 170-175.

Sáinz de Baranda, P., Rodríguez, P.L., Santonja, F., López, P.A., Andújar, P.; Ferrer, V. \& Pastor, A. (2006) Effects of hamstring stretching exercises on the toe-touch test in elementary schoolchildren. Journal of human movement studies, 51 (4), 277-289.

Sánchez Rivas, E.; Mayorga-Vega, D.; Fernández Rodríguez, E.; Merino-Marban, R. (2014). Effect of a hamstring stretching programme during physical education lessons in primary education. Journal of Sport and Health Research, 6(2), 159-168.

Santonja Medina, F.; Sainz de Baranda, P.; Rodríguez, P.L; López Miñarro, P.A; Canteras Jordana, M. (2007). Effects of frequency of static stretching on straight-leg raise in elementary school children. The Journal of Sports Medicine and Physical Fitness, 47, 304-308.

\section{Notas}

[1] Máster Universitario en Investigación en Educación Física y Salud

[2] Doctor en Ciencias de la Actividad Física y el Deporte

\section{BY-NC-ND}

